\title{
Magnetic drug delivery with FePd nanowires
}

\author{
Kirsten M. Pondman ${ }^{a}$, Nathan D. Bunt ${ }^{a}$, A. Wouter Maijenburg ${ }^{b}$, Richard J.A. van Wezel ${ }^{c}$, \\ Uday Kishore ${ }^{\mathrm{d}}$, Leon Abelmann ${ }^{\mathrm{e}}$, Johan E. ten Elshof ${ }^{\mathrm{b}}$, Bennie ten Haken ${ }^{\mathrm{a}, *}$ \\ a Neuro Imaging, MIRA Institute, University of Twente, Enschede, The Netherlands \\ ${ }^{\mathrm{b}}$ Inorganic Material Science, MESA + Institute for Nanotechnology, University of Twente, Enschede, The Netherlands \\ c Biomedical Signals and Systems, MIRA, Twente University, Enschede, The Netherlands \\ d Centre for Infection, Immunity and Disease Mechanisms, Biosciences, Brunel University, London, UK \\ e Transducer Science and Technology group, MESA + Institute for nanotechnology, University of Twente, Enschede, The Netherlands
}

\section{A R T I C L E I N F O}

\section{Article history:}

Received 29 June 2014

Received in revised form

16 October 2014

Accepted 18 October 2014

\section{Keywords:}

Magnetic drug delivery

Nanowire

Toxicity

Immune response

\begin{abstract}
A B S T R A C T
Magnetic drug delivery is a promising method to target a drug to a diseased area while reducing negative side effects caused by systemic administration of drugs. In magnetic drug delivery a therapeutic agent is coupled to a magnetic nanoparticle. The particles are injected and at the target location withdrawn from blood flow by a magnetic field. In this study a FePd nanowire is developed with optimised properties for magnetic targeting. The nanowires have a high magnetic moment to reduce the field gradient needed to capture them with a magnet. The dimensions and the materials of the nanowire and coating are such that they are dispersable in aqueous media, non-cytotoxic, easily phagocytosed and not complement activating. This is established in several in-vitro tests with macrophage and endothelial cell lines.

Along with the nanowires a magnet is designed, optimised for capture of the nanowires from the blood flow in the hind leg of a rat. The system is used in a pilot scale in-vivo experiment. No negative side effects from injection of the nanowires were found within the limited time span of the experiment. In this first pilot experiment no nanowires were found to be targeted by the magnet, or in the liver, kidneys or spleen, most likely the particles were removed during the fixation procedure.
\end{abstract}

(c) 2014 Published by Elsevier B.V.

\section{Introduction}

Amongst many receptor dependent drug targeting methods with nanoparticles, magnetic drug delivery is a promising physical method to target pharmaceuticals. In this method, a drug is coupled to a magnetic particle and injected into blood flow. A magnet located close to the target location is used to capture the magnetic particles and therapeutics in the target area. The method is promising in modelling studies and some results in animals and a single clinical test have been published, but no magnetic drug delivery applications are used in clinics today [1-9]. The majority of magnetic particles used in biomedical applications are the socalled SPIONs (superparamagnetic iron oxide nanoparticles), which consist of one or multiple magnetite or maghemite cores with a biocompatible coating and active ligands [10]. The small magnetic moment of these particles makes it challenging to retain particles at the target site while withstanding the drag of blood flow. To overcome the drag force the magnetic field and gradient have to be extremely large, or the particles have to agglomerate

\footnotetext{
* Corresponding author.

E-mail address: b.tenhaken@utwente.nl (B. ten Haken).
}

into bundles; often causing embolisation of the vessels. Nanowires, with their elongated shape and anisotropic physical and magnetic properties, can be used to overcome some of the limitations of SPIONs in in-vivo targeting applications. Magnetic nanorods and nanowires are therefore receiving growing attention in biomedicine [11-16]. While the small diameter of nanowires makes it possible for the particle to pass through the narrow capillaries, the remnant magnetisation increases the range and effectiveness of magnetic interactions at a distance from external magnets. This allows magnetic drug targeting at locations deeper inside the body [12]. Whereas spherical particles only respond to magnetic flux gradients, high aspect ratio particles allow the application of torque with a relatively weak external field to perform dynamic targeted cell therapy [13]. Due to their increased surface to volume ratio, more drugs and additional targeting ligands (e.g. ligands aiding the endocytosis of the particles) can be bound.

In this study the potential of rod-like, magnetic nanoparticles for magnetic drug delivery is investigated. First the magnetic properties of elongated nanoparticles are determined, then a magnetic nanowire is developed with optimised characteristics for magnetic drug delivery. FePd nanowires were prepared by electrodeposition. This alloy was chosen, because it is highly magnetic, non-toxic and not prone to oxidation [17]. Besides the magnetic 
force on the particles, the effectiveness of nanoparticles as intravenous drug delivery platforms is strongly influenced by rapid elimination of the particles from the systemic circulation by the immune cells [18]. Therefore initial tests were performed to analyse the activation of the innate immune systems complement system by the developed particles. The innate immune system plays a key role in protection against pathogens and synthetic particles including nanoparticles [19-23,18]. When a particle is recognised by the innate immune complement system, it will be marked for uptake by phagocytic cells (opsonised) and unable to reach its target [24]. For magnetic drug delivery not only the particles, but also the external magnet has to be specially designed with optimised characteristics for the trapping of the particles. Here, we designed a magnet able to capture the developed nanowires from capillary blood flow, and of which the dimensions, field and gradient can be scaled up for application in a clinical setting. Finally the magnet-nanowire system is applied in a pilot in-vivo magnetic drug delivery test.

\section{Theory}

\subsection{Magnetic properties of nanoparticles}

In superparamagnetic particles thermal fluctuations allow the magnetisation of a magnetic particle to cross over the energy barrier between stable directions of magnetisation. The time it takes to flip the magnetisation is given by the Neel-Arrhenius equation:

$\tau^{-1}=f_{0} e^{-E_{B} / k_{B} T}$

with attempt frequency $f_{0}=10^{-9}-10^{-10} \mathrm{~s}^{-1}$ and $E_{B}$ the energy barrier. For single domain ellipsoids, in which the magnetisation reverses through coherent rotation, that are well dispersed in a carrier medium the energy barrier is determined by the shape $E_{S}$ and crystalline anisotropy energy $E_{c a}$. The crystalline anisotropy originates exclusively from intrinsic material properties:

$\boldsymbol{E}_{\mathrm{ca}}=\boldsymbol{K}_{\mathrm{ca}} V$

For spherical particles the orientation of the particle in the field has no influence on the magnetisation direction, therefore there is no shape anisotropy and crystalline anisotropy is the dominating anisotropy factor.

Elongated particles are more easily magnetised along the long axis. This shape anisotropy energy can be described with

$\boldsymbol{E}_{\mathbf{S}}=\frac{1}{2} \mu_{0}\left(N_{a} M_{a}^{2}+N_{b} M_{b}^{2}+N_{c} M_{c}^{2}\right) V$

For a prolate ellipsoid with axis $c>a=b$, with aspect ratio $k=c / a$, the demagnetisation factors $N_{a}, N_{b}$ and $N_{c}$ are given by $[25,26]$

$$
\begin{aligned}
N_{a} & =N_{b} \\
& =\frac{k}{2\left(k^{2}-1\right)}\left[k-\frac{k}{2 \sqrt{k^{2}-1}} \ln \left(\frac{k+\sqrt{k^{2}-1}}{k-\sqrt{k^{2}-1}}\right)\right] N_{c} \\
& =1-2 N_{a}
\end{aligned}
$$

The shape anisotropy difference between the two axes is described by anisotropy constant:

$\boldsymbol{K}_{\mathbf{S}}=\frac{1}{2} \mu_{0}\left(3 N_{a}-1\right) M_{s}^{2}$

In elongated particles, the crystal anisotropy is negligible compared to the shape anisotropy. The energy barrier that has to be overcome to flip the magnetisation is

$E_{\mathbf{B}}=\mathbf{K V}$

For spherical particles $K$ will be the crystalline anisotropy constant $\left(K_{c a}\right)$, for elongated particles the shape anisotropy constant $\left(K_{s}\right)$. Within the time constant of magnetic drug delivery we consider particles superparamagnetic if they change their magnetisation direction within one second $(\tau \simeq 1 \mathrm{~s})$. The maximum volume at which the particle is superparamagnetic is:

$V_{\max }=\frac{25 k_{B} T}{K_{s}}$

At high aspect ratio $k>10, N c \simeq 0$ :

$V_{\text {max }}=\frac{25 k_{B} T}{\frac{1}{4} \mu_{0} M_{s}^{2}}$

which for magnetite will give a maximum nanowire volume of approximately $2 \times 10^{-24} \mathrm{~m}^{3}\left(2000 \mathrm{~nm}^{3}\right)$. All particles with a volume larger than $V_{\max }$ as given by Eq. (7) are ferromagnetic particles. Note that this is an upper limit, derived under the condition that the particle reverses coherently. For very long nanowires this might not be the case.

The size of single domain ferromagnetic particles is limited by the magnetic self-energy which to avoid formation of a multi domain state has to be lower than the domain wall energy given by

$\boldsymbol{E}_{\mathrm{DW}}=2 \pi \sqrt{\mathbf{A K}} \cdot \pi \mathrm{ac}$

with $A$ being the exchange stiffness constant of the material, the critical diameter is around $40 \mathrm{~nm}$ for spherical magnetite particles and largest for elongated particles $(1 \mu \mathrm{m})$.

\subsection{Forces on magnetic particles in blood flow}

In the vascular system, the blood flow exerts a force on the particles dragging them along with the flow. Magnetic targeting will take place in the capillary system, where blood flow can be modelled by a slow Newtonian non-pulsating flow. The targeting is analysed by the first order forces only, which are the magnetic force $\left(\boldsymbol{F}_{m}\right)$ and the viscous drag force $\left(\boldsymbol{F}_{d}\right)$. Due to the small size and mass of the nanoparticles all second order forces such as gravitational and buoyancy forces, and inertia are neglected.

\subsubsection{Magnetic force}

The magnetic force $\boldsymbol{F}_{m}$ on a small particle due to an external magnetic flux $\boldsymbol{B}$ can be assumed to be equal to the force experienced by a point-like magnetic dipole [10,27]. The magnetic force is defined by

$\boldsymbol{F}_{m}=\nabla\left(\boldsymbol{m}_{p} \cdot \boldsymbol{B}\right)=\nabla\left(\boldsymbol{m}_{p} \cdot \mu_{f} \boldsymbol{H}_{a}\right)$

where $\mu_{f}$ is the permeability of blood. The magnetic dipole moment of the particle, $m_{p}$, depends on the magnetisation and the volume:

$m_{p}=V_{p} M$

For ferromagnetic particles $M$ in this equation is equal to the saturation magnetisation of the particle $M_{s}$. For superparamagnetic particles the magnetisation depends on the local magnetic field intensity $\left(\boldsymbol{H}_{i n}\right)$, the field inside the particle, which differs from the applied field $\left(\boldsymbol{H}_{a}\right)$ due to the demagnetisation field $\left(\boldsymbol{H}_{d}\right)$ that opposes $\boldsymbol{H}_{a}: \boldsymbol{H}_{i n}=\boldsymbol{H}_{a}-\boldsymbol{H}_{d} . \boldsymbol{H}_{d}$ depends on $M$ and the demagnetisation factor $(N)$ which in turn depends on the shape of the particle:

$H_{\mathrm{d}, z}=N_{x} M_{x}$ 
Here $N_{x}$ is the demagnetisation factor along the $z$-direction. To minimise the Zeeman energy the particle will align with the magnetic field, therefore there is only one demagnetisation factor for every particle, and the internal field strength and magnetisation can be determined:

$M=M_{s} \mathcal{L}\left(\frac{M \mu_{0} \boldsymbol{H}_{i n}}{k_{B} T}\right)$

with $M_{s}$ being the saturation magnetisation of the material and $\mathcal{L}$ being the Langevin function.

\subsubsection{Drag force}

The fluidic drag force can be calculated using Stokes' law for drag on a sphere in a laminar flow field with Reynolds number smaller than 1, which is a valid approximation for the flow in the microvasculature:

$\boldsymbol{F}_{d}=-6 \eta r_{p}\left(\boldsymbol{v}_{p}-\boldsymbol{v}_{f}\right)$

In this equation $\eta$ represents the viscosity of the medium $\left(\eta_{\text {plasma }}=1.2 \times 10^{-3} \mathrm{~Pa} \mathrm{~s}\right), v_{p}$ and $v_{f}$ are the velocity of the nanoparticle and the medium respectively. For prolate ellipsoids Eq. (14) can be adapted for their specific shape anisotropy [28,29]:

$\boldsymbol{F}_{d}=-\eta \pi a\left(v_{p}-v_{f}\right) K_{d}$

With shape factor

$K_{d}=\frac{4}{\ln (2 k)-\frac{1}{2}}$

when the particle is moving in the direction of the long axis and

$K_{d}=\frac{8}{\ln (2 k)+\frac{1}{2}}$

when the particle is moving perpendicular to the direction of the long axis.

\section{Methods and materials}

\subsection{FePd nanowires}

FePd nanowires were synthesised by a method adapted from [30-32] by templated electrodeposition in a conventional threeelectrode setup. First, an Au layer was sputtered onto a PCTE membrane with a pore size of $50 \mathrm{~nm}$ using a Perkin-Elmer 2400 sputtering system under argon atmosphere. The Au layer, isolated with a glass plate, was used as working electrode in the electrochemical deposition process. A small Pt mesh was used as counter electrode and $\mathrm{Ag} / \mathrm{AgCl}$ in $3 \mathrm{M} \mathrm{KCl}$ (Metrohm Autolab) as reference electrode. All reported potentials are with respect to the reference electrode. The electrodes were connected to a potentiostat ( $\mathrm{Au}$ tolab PGSTAT 128N from Metrohm Autolab, The Netherlands). For electrodeposition of nanowires, the electrodes were placed inside a plating solution containing $0.1 \mathrm{M}$ Iron(III)sulfate pentahydrate $\left(\mathrm{Fe}_{2}\left(\mathrm{SO}_{4}\right) 3 \cdot 5 \mathrm{H}_{2} \mathrm{O}\right)$ (Acros organics, $97 \%$ ), $0.02 \mathrm{M}$ tetraamminepalladium(II)chloride monohydrate $\left(\mathrm{Pd}\left(\mathrm{NH}_{3}\right)_{4} \mathrm{Cl}_{2} \cdot \mathrm{H}_{2} \mathrm{O}\right)(97 \%$ Alfa Aesar), 0.3 M 5-sulfosalicylic acid (99\%, Alfa Aesar) and $0.3 \mathrm{M}$ ammonium sulphate $\left(\left(\mathrm{NH}_{4}\right)_{2} \mathrm{SO}_{4}\right)(99 \%$, Sigma Aldrich) at $\mathrm{pH}$ 5.0. Nanowires were formed by pulsed electrodeposition, using 4 cycles of $50 \mathrm{~s}$ at a voltage of $-1.1 \mathrm{~V}$ followed by $150 \mathrm{~s}$ at a voltage of +0.1 V. Pulsed deposition was used to allow for both the Pd and the less noble Fe to deposit. After deposition, the membranes were dissolved in dichloromethane and free nanowires washed at least 3 times in dichloromethane and 3 times in milliQ water by centrifugation.

\subsection{Biofunctionalisation}

The functionalisation of the FePd nanowires was accomplished by a method inspired by [33]. FePd nanowires at $100 \mu \mathrm{g} / \mathrm{ml}$ were sonicated in hexane with $10 \mu \mathrm{g} / \mathrm{ml}$ oleic acid (OA) for $30 \mathrm{~min}$, excess OA was removed by centrifugal washing (2000 g) in hexane. From this point FePds were handled in sterile conditions and sterile solutions were used. Hydrophilic coating was achieved by adding $0.5 \mathrm{ml}$ of $10 \mathrm{mg} / \mathrm{ml} \mathrm{FePd-OA}$ in hexane to $20 \mathrm{ml}$ of $0.5 \mathrm{mg} /$ ml Pluronic-F108 (PF) (Sigma Aldrich) in $\mathrm{H}_{2} \mathrm{O}$. By water bath sonication a dispersion was obtained, subsequently hexane was evaporated. PF was allowed to adhere overnight under stirring. Excess Pluronic-F108 was removed by washing (2000 g) three times in sterile $\mathrm{H}_{2} \mathrm{O}$. To label the FePd fluorescent Nile red (NR) (Sigma Aldrich) was dissolved in $1 \mathrm{mg} / \mathrm{ml}$ in DMSO and $100 \mu \mathrm{l}$ was added to $10 \mathrm{ml}$ of FePd-OA-PF at $1 \mathrm{mg} / \mathrm{ml}$ in $\mathrm{H}_{2} \mathrm{O}$. To allow partitioning of the NR into the OA layer surrounding the FePd the solution was stirred overnight. FePd were washed five times in sterile $\mathrm{H}_{2} \mathrm{O}$ followed by two washings in sterile PBS (Sigma Aldrich). Labelling was analysed by fluorescence microscopy.

\subsection{Characterisation}

Analysis of the nanowires was performed with a Zeiss HR-LEO 1550 FEG Scanning Electron Microscope (SEM) operating at $2.0 \mathrm{kV}$. Transmission electron microscopy (TEM) was performed on a Philips CM300ST-FEG Transmission Electron Microscope. Magnetic characterisation was done using a vibrating sample magnetometer (Model 10 Mark II VSM, Microsense). Samples were either measured in the synthesis membrane or dried in a glass container before measurement; a blank measurement of the empty substrate was subtracted from the sample data.

\subsection{Complement activation and consumption assay for the classical pathway}

FePd nanowire suspensions $(100 \mu \mathrm{l}$ of $1 \mathrm{mg} / \mathrm{ml}, 0.5 \mathrm{mg} / \mathrm{ml}$, $0.25 \mathrm{mg} / \mathrm{ml}$ and $0.125 \mathrm{mg} / \mathrm{ml}$ ) in PBS were added to $100 \mu \mathrm{l}$ of $1: 1$ diluted human serum in DGVB ${ }^{++}$. Zymosan $(0.2 \mathrm{mg}$ in $100 \mu \mathrm{l} \mathrm{PBS}$; Sigma) served as a positive control. The samples were incubated for $1 \mathrm{~h}$ at $37^{\circ} \mathrm{C}$ with occasional shaking, followed by centrifugation to remove the nanowires $(13,000 \mathrm{~g}, 10 \mathrm{~min})$.

To investigate whether FePd nanowires activate (consume) complement in human serum, the sera's capacity to lyse antibodysensitised sheep erythrocytes (EA) was tested. EA were prepared [19] using sheep erythrocytes from TCS Biosciences and stored in dextrose gelatin veronal buffer $\left(\mathrm{DGVB}^{++}: 2.5 \mathrm{mM}\right.$ sodium barbital, $71 \mathrm{mM} \mathrm{NaCl}, 0.15 \mathrm{mM} \mathrm{CaCl}_{2}, 0.5 \mathrm{mM} \mathrm{MgCl}_{2}, 2.5 \% \mathrm{w} / \mathrm{v}$ glucose, $0.1 \% \mathrm{w} / \mathrm{v}$ gelatin, $\mathrm{pH} 7.4$ ) at $10^{9}$ cells $/ \mathrm{ml}$. The serum supernatants of each sample were two-fold serially diluted (1/10 to $1 / 5120$ in $\mathrm{DGVB}^{++}$) and placed in microtitre wells. $100 \mu \mathrm{l}$ of each dilution was incubated with $100 \mu \mathrm{l}$ of EA $\left(10^{8}\right.$ cells $/ \mathrm{ml}$ in $\left.\mathrm{DGVB}^{++}\right)$in U-shaped wells (Fisher Scientific) for $1 \mathrm{~h}$ at $37^{\circ} \mathrm{C}$. Thereafter, EA were spun down $(1000 \mathrm{~g}, 10 \mathrm{~min}, \mathrm{RT})$, and the released haemoglobin in $150 \mu \mathrm{l}$ of each supernatant was read at $541 \mathrm{~nm}$. Total haemolysis (100\%) was measured by lysing EA with water. Background spontaneous haemolysis $(0 \%)$ was determined by incubating EA with buffer only. $\mathrm{CH} 50$ values, which correspond to the dilution factor of the serum that results in 50\% cell lysis, were calculated and compared. All assays were performed in triplicates.

\subsection{Confocal imaging}

A Zeiss (Göttingen, Germany) LSM 510 confocal laser scanning microscope (CLSM) was used to image uptake of FePd nanowires by cells in bright field. $10^{4}$ RAW264.7 or HeLa cells per well were 
incubated in $100 \mu \mathrm{l}$ DMEM containing $10 \%$ heat inactivated FCS, $2 \mathrm{mM}$ L-glutamine, $100 \mathrm{U} / \mathrm{ml}$ of penicillin and $100 \mu \mathrm{g} / \mathrm{ml}$ of streptomycin and $1 \mathrm{mM}$ of sodium pyruvatein a 96-well plate. Cells were allowed to adhere for $2 \mathrm{~h}$ after which FePd nanowires were added with a nanowire concentration of approximately 10 nanowires/cell. This concentration was estimated by assuming that the nanowires have the density of bulk FePd and average dimensions as estimated from SEM and TEM images. With ICP-MS (Inductively coupled plasma mass spectrometry) (data not shown) we found a Fe:Pd ratio of 50:50, which gives an approximate alloy density of $10^{7} \mathrm{~g} / \mathrm{m}^{3}$. Control cell samples contained only PBS and no nanowires. Images were obtained after $24 \mathrm{~h}$ incubation at $37^{\circ} \mathrm{C}$ in $5 \%$ $\mathrm{CO}_{2}$ atmosphere.

\subsection{Dose dependent cell viability on phagocytotic and non-phago- cytotic cells}

For viability testing HeLa (a human cervical carninoma epithelial cell line) and RAW264.7 (a murine macrophage cell line) were cultured in DMEM containing 10\% heat inactivated FCS, $2 \mathrm{mM}$ L-glutamine, $100 \mathrm{U} / \mathrm{ml}$ of penicillin and $100 \mu \mathrm{g} / \mathrm{ml}$ of streptomycin and $1 \mathrm{mM}$ of sodium pyruvate. Adherent cells, RAW264.7 and HeLa, were seeded on 96-well culture plates at $5 \times 10^{3}$ cells/ well in $100 \mu \mathrm{l}$ of complete cell medium. Cells were allowed to adhere to the plate for $24 \mathrm{~h}$ at $37^{\circ} \mathrm{C}$ in $5 \% \mathrm{CO}_{2}$ atmosphere. Incubation of non-fluorescent labelled FePd nanowires was done by adding $100 \mu \mathrm{l}$ of FePd nanowire dispersion in PBS to the cells, for $48 \mathrm{~h}$ at $37{ }^{\circ} \mathrm{C}$ in $5 \% \mathrm{CO}_{2}$ atmosphere. The final concentrations of the FePd nanowires were 1, 10, 100 and 1000 nanowires/cell. All experiments were performed in triplicates. After $48 \mathrm{~h}$ the cell medium was carefully removed and the cells were washed with PBS, thereafter $100 \mu \mathrm{l}$ of cell medium was added with $10 \mu \mathrm{l}$ of CellTiter Blue and incubated for $4 \mathrm{~h}$. The fluorescence was measured on a spectrophotometer (Victor, Perkin Elmer) with an excitation wavelength of $560 \mathrm{~nm}$ and an emission wavelength of $590 \mathrm{~nm}$. The fluorescence values were normalised by the controls and expressed as percent viability. Validity of the assay was assessed by cell counting and visual inspection of the cells.

\subsection{In-vivo magnetic drug delivery}

The animal research protocol was approved by the animal ethics committee of the de Radboud University Nijmegen. Four male Wistar rats (6-8 weeks), body weight $260 \pm 10 \mathrm{~g}$, were used for the studies. Animals were housed and fed according to local regulations.

Rats were kept under anesthesia with $2-3 \%$ isoflurane during the entire procedure. The animals were placed ventrally on a platform with the right hind leg between the poles of the magnet (Fig. 1). Nanowire suspensions in PBS were injected via the tail vein at $10 \mathrm{mg} / \mathrm{kg}$. The magnet was switched on $30 \mathrm{~s}$ after the injection to allow for distribution of the particles and the rats were retained in the magnet for $30 \mathrm{~min}$. Thereafter, the animals were fixated by vascular perfusion through the heart with paraformaldehyde. Hind legs, liver, spleen and kidneys of the rats were sectioned, according to anatomical structures and tissue samples were measured for detection of FePd nanowires with a vibrating sample magnetometer (Quantum Design, San Diego, CA, USA) with a variable magnetic field of $\pm 4 \mathrm{~T}$. Tissue samples were kept inside quartz NMR tubes and fixated to ensure that no movement occurred owing to the vibrations of the device, as described in [34]. In similar experiments using Endorem particles a lower detection limit of $1.5 \mu \mathrm{g}$ of iron with an accuracy of $0.5 \mu \mathrm{g}$ was found [34], and similar results were found with a range of FePd nanowires dispersed in water and agar (results not shown).

\section{Results and discussion}

\subsection{FePd nanowires}

FePd nanowires were made with tunable length depending on the number of voltage cycles $(50 \mathrm{~s}-1.1 \mathrm{~V}$ and $150 \mathrm{~s}+0.1 \mathrm{~V}$ ) used in the electrodeposition. Using 4 cycles nanowires were made with length $1.9 \pm 0.3 \mu \mathrm{m}$ (SEM 100 wires) and diameter $88 \pm 15 \mathrm{~nm}$ (TEM 100 wires) (Fig. 2) leading to an aspect ratio of 22. With their volume of $10^{-20} \mathrm{~m}^{3}$ the nanowires are not small enough to be considered as superparamagnetic. The magnetisation curves in Fig. 3a show the room temperature magnetisation curves for uncoated FePd nanowires inside the template with magnetic field $(H)$ applied in three different directions, 0,45 and $90^{\circ}$ to the wire direction. The squareness ratio $M_{r} / M_{s}$ and coercivity are known to increase significantly with aspect ratio [35]. In the case of the FePd wires the obtained hysteresis loops cannot be explained in terms of pure coherent rotation or superparamagnetic behaviour. The curves show remanence in all directions, as can be seen from the nearly equal squareness ratio $M_{r} / M_{s}=0.13 \pm 0.2$ in all directions. Moreover, the sample is easier to saturate in the hard-axis direction $\left(90^{\circ}\right)$. The angular behaviour is therefore opposite to what is expected from a simple coherent rotation model, or even more advanced micromagnetic models and experiments on Co nanowires of $50 \mathrm{~nm}$ diameter spaced by $150 \mathrm{~nm}$ [36]. Similar observations have however been made for $\mathrm{Co}_{75} \mathrm{Ni}_{25}$ wires [37]. At a diameter of $35 \mathrm{~nm}$ hard axis loops have lower coercity and remanence, and take longer to saturate. At $65 \mathrm{~nm}$ however, the loops are similar to our observations. The authors attribute this effect to an increased contribution of crystalline anisotropy perpendicular to the wire, which balances the shape anisotropy. Indeed, also our wires are strongly polycrystalline, as is supported by the TEM image and XRD analysis. The VSM loops of randomly oriented
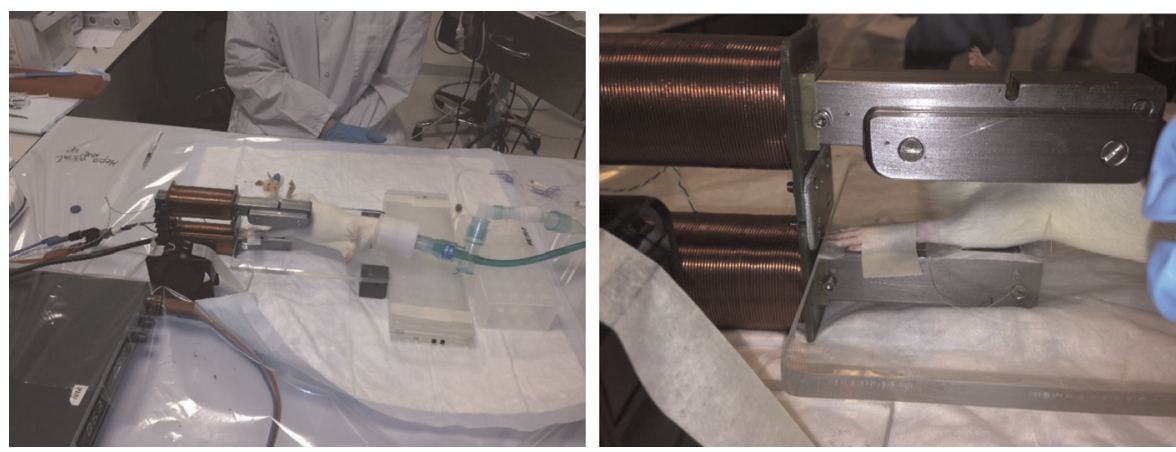

Fig. 1. Set-up in-vivo magnetic drug delivery. 
a

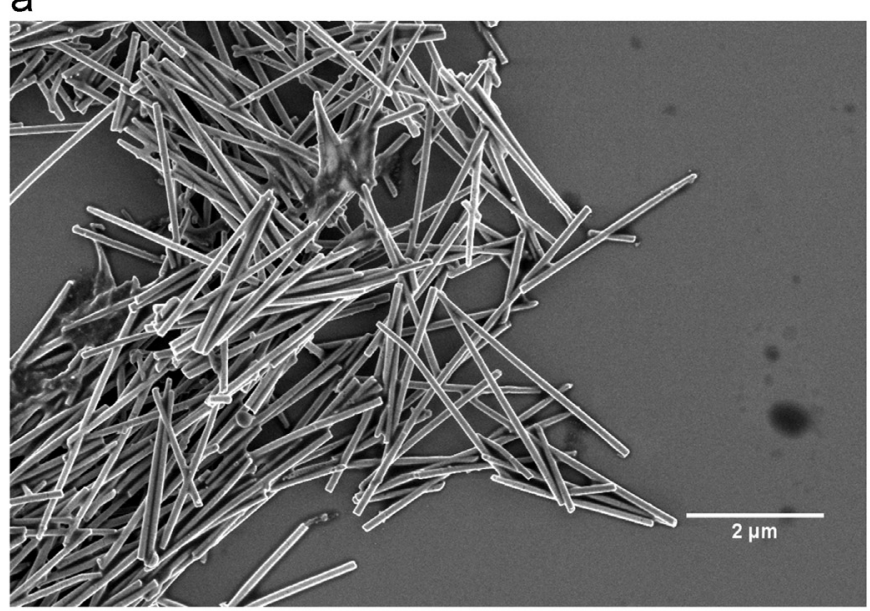

C

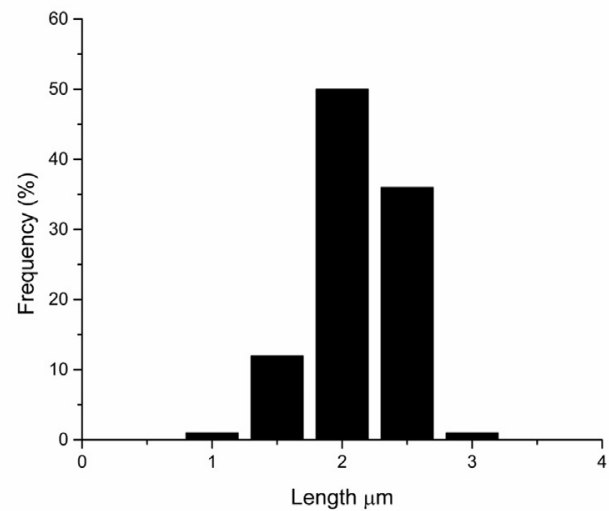

b

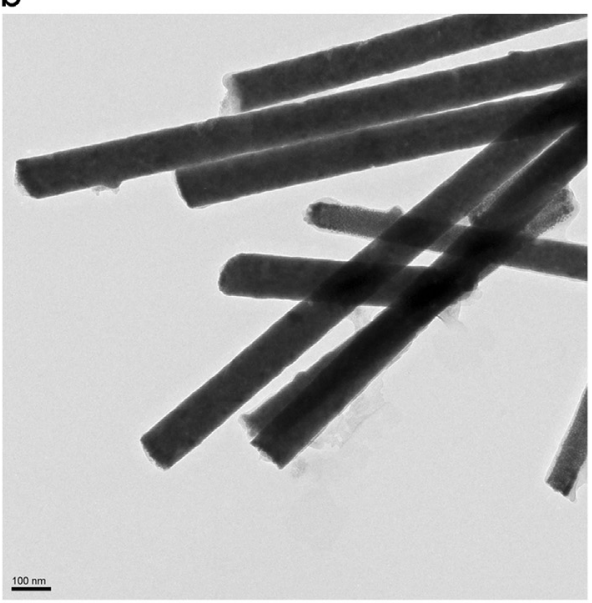

d

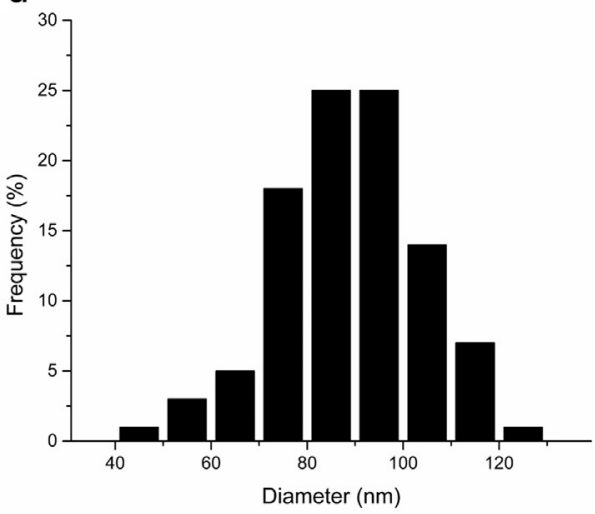

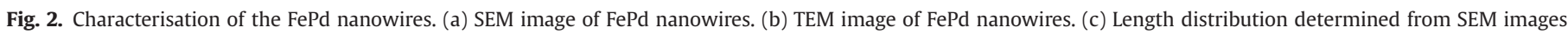
$(n=100)$. (d) Diameter distribution determined from TEM images $(n=100)$.

nanowires are shown in Fig. 3. With respect to the array, the remanence has increased to 0.3 , which supports the assumption of a strong transverse crystal anisotropy if one assumes that the magnetic easy axis of the wires is perpendicular to their long axis. The coercivity strongly decreased to $8 \mathrm{kA} / \mathrm{m}$, which might be attributed to the fact that the wires become superparamagnetic. All this of course is highly speculative, and will require further magnetic characterisation.

\section{a}

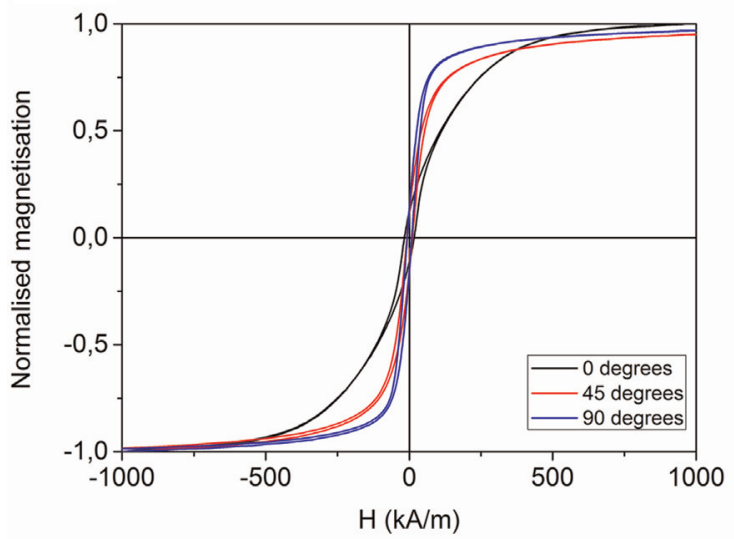

\subsection{Magnet design}

For a magnet to be able to trap particles from the blood flow in capillaries the magnetic force on the particle has to be larger than the drag force. The drag force is mainly determined by the particles orientation and the blood flow velocity, which in small capillaries is around $1 \mathrm{~mm} / \mathrm{s}$ [38]. The particles can be orientated freely in the blood flow although it is most likely that the particles

b

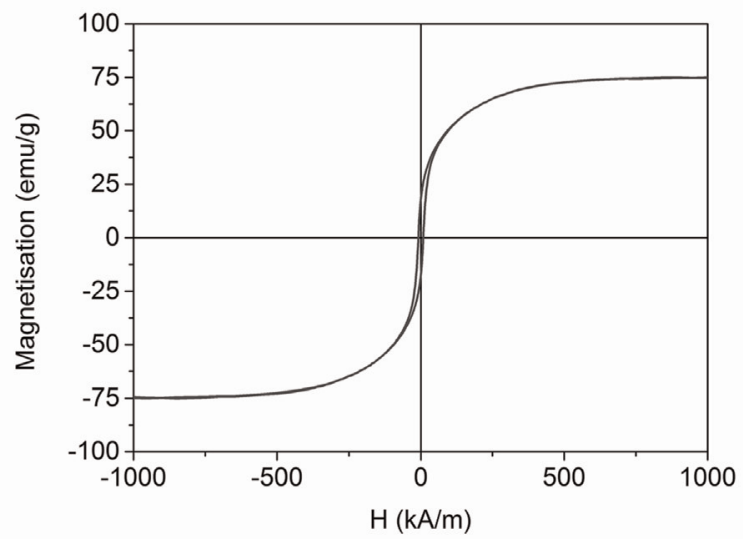

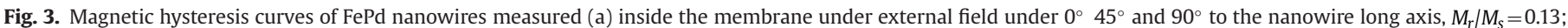
(b) in random orientation with $M_{s}=80 \mathrm{emu} / \mathrm{g}, M_{r}=25 \mathrm{emu} / \mathrm{g}$ and $H_{c}=8 \mathrm{kA} / \mathrm{m}$. 
will align their long axis with the flow, especially when the torque caused by the magnet helps us to align in this orientation. In a "worst case scenario" the particle will flow perpendicular to the blood flow away from the magnet which tries to capture it. For the FePd nanowires with the dimensions and characteristics described above, the drag force is $6.7 \mathrm{pN}$ in this orientation. When the particle is flowing parallel to the vessel the drag force is $4.4 \mathrm{pN}$. Using this setup, without the use of superconducting elements it is possible to obtain a maximum magnetic field strength of approximately $50 \mathrm{mT}(\cong 400 \mathrm{kA} / \mathrm{m})$ in the entire field where this is required. With the FePd density found above, we can estimate the particle magnetic moment: $10^{-15} \mathrm{~A} \mathrm{~m}^{2}$. To achieve capture of these particles a magnetic field gradient of at least $0.83 \mathrm{~T} / \mathrm{m}$ is needed in the perpendicular orientation and $0.55 \mathrm{~T} / \mathrm{m}$ in the parallel orientation.

The magnet was developed for application in the in-vivo animal experiment (rat), with an additional requirement that the design should be scalable for use in a clinical application e.g. for application in breast cancer. To be able to control the field strength and the gradient a U shaped electromagnet was developed with an iron core. The gradient was obtained by using different pole sizes on either side. The inter-pole distance is set to $3 \mathrm{~cm}$ which is the approximate size of a rat's hind leg. To avoid loss of field strength at the edges the corners of the magnet were rounded. The final design of the magnet is depicted in Fig. 4(a) and (b). The magnet core is $2 \mathrm{~cm}$ thick pure iron (coated to avoid oxidation), around the two arms a coil was wounded, each 480 turns in 6 layers coated with Stycast-1266 to increase heat conduction. Using a 2.5 A current the magnet had a center of gap magnet field of $70 \mathrm{mT}$ and a gradient up to $2.4 \mathrm{~T} / \mathrm{m}$ (measured using a Hall effect sensor CYHS80) (Fig. 4c). These values exceed the required calculated values, as a precaution to ensure success of this first pilot experiment. In an initial test with nanowires in a tube the FePd nanowires were easily attracted by the magnet, while in similar conditions SPIOs (Chemicell, $100 \mathrm{~nm}$ ) could not be attracted. This indicates the added value of the FePd nanowires in magnetic drug delivery.

\subsection{In-vitro and in-vivo results}

The uptake of FePd nanowires was studied by confocal microscopy (Fig. 5). After $24 \mathrm{~h}$ of incubation, both RAW264.7 and HeLa cells had taken up many nanowires and clusters of nanowires. RAW264.7 cells had taken up significantly more nanowires than HeLa. As RAW264.7 cells are macrophages, the uptake pathway in these cells is most likely phagocytosis. Confocal fluorescent microscopy analysis showed that the nanowires were completely taken up in the cytoplasm of these cells without signs of frustrated phagocytosis (data not shown). This indicates that macrophages will be able to remove the particles from the bloodstream within a $24 \mathrm{~h}$ period. In a CTB viability assay on both types of cells with 1 , 10,100 and 1000 nanowires per cell no significant reduction of viability was observed (Fig. 5e) indicating the low toxicity of the nanoparticles.

Besides toxicity also the response of the immune system was taken into account. Activation of the complement system can lead to opsonisation of the particles, labelling them for rapid removal from the circulation by macrophages (especially in the liver). No complement activation was found even by the high $(1 \mathrm{mg} / \mathrm{ml})$ concentration of FePd nanowires (data not shown).

In the in-vivo test, the rats showed no negative side effects after the injection of the FePd nanowires compared to a control experiment with PBS only. In VSM measurements no magnetic signal could be detected in the liver, spleen and kidneys of the rats, indicating that the immune response and first pass filtration of the kidneys did not eliminate the FePd from circulation. For most SPIO particles localisation in the kidneys can be detected within a a

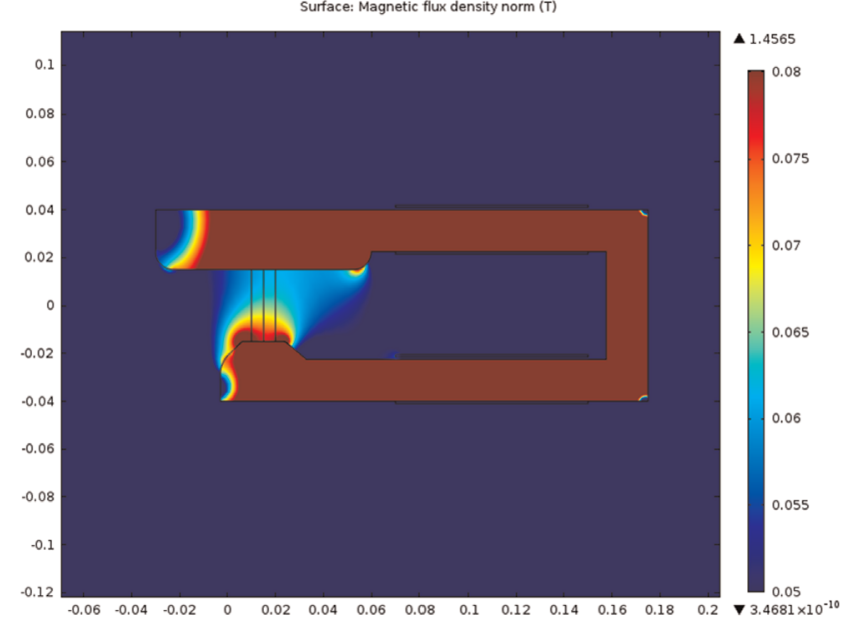

b

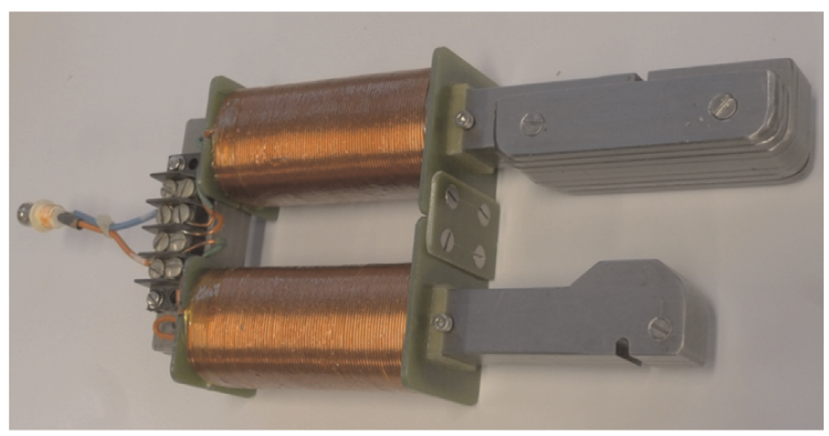

C

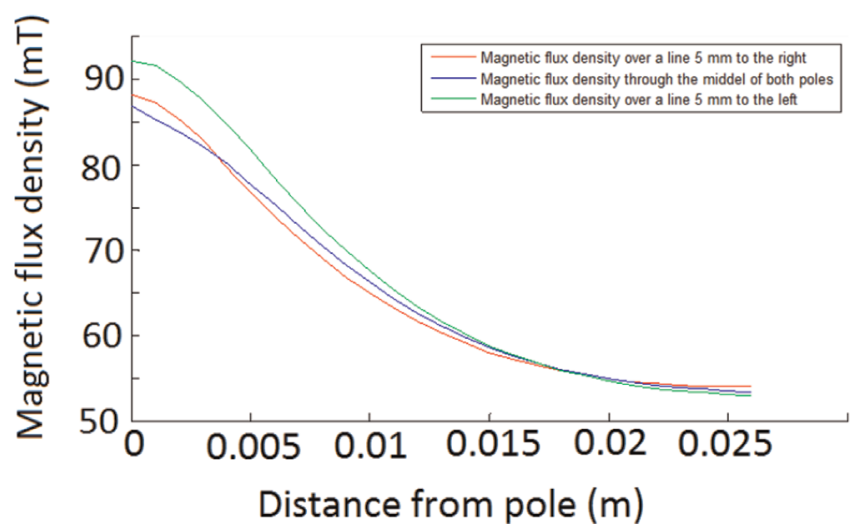

Fig. 4. Magnet design and characterisation. (a) Magnet design in COMSOL indication flux densities. (b) Picture of final magnet. (c) Measured magnetic field strength at the locations indicated in (a).

30 min period after injection [39]. This could indicate that the FePd nanowires are not rapidly recognised by the immune system and remain in circulation. In the targeted hind leg of the rat, no significant or quantifiable signal could be measured, which indicates that less than $1.5 \mu \mathrm{g}$ of iron was present in the samples. Excretion of the particles is unlikely during 30 minutes of the experiments. No attempts were made to find the FePd nanowires using microscopy. 
a

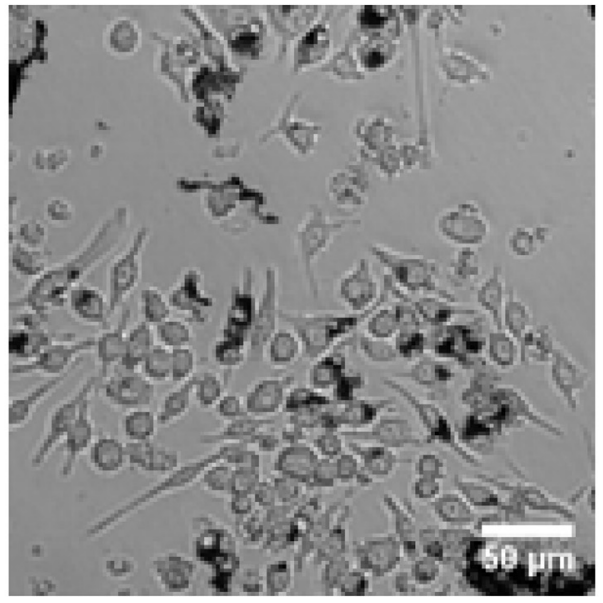

C
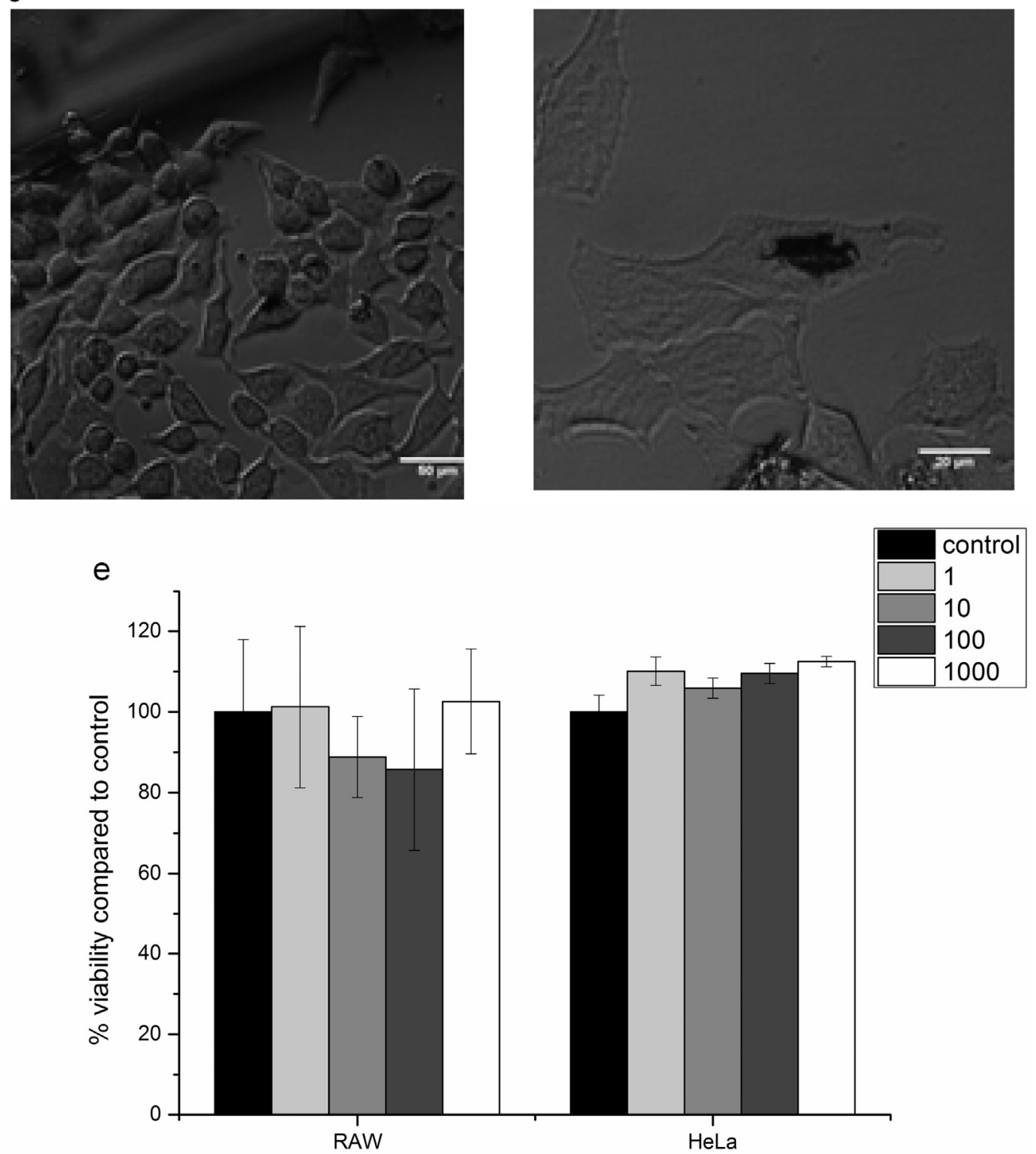

b

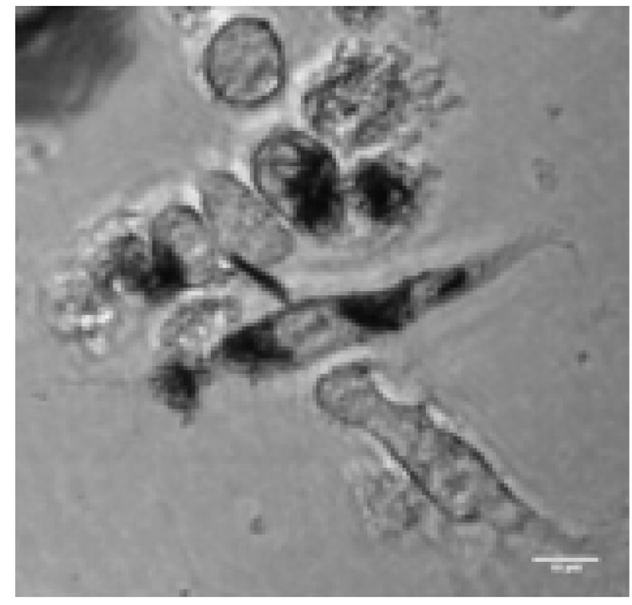

d

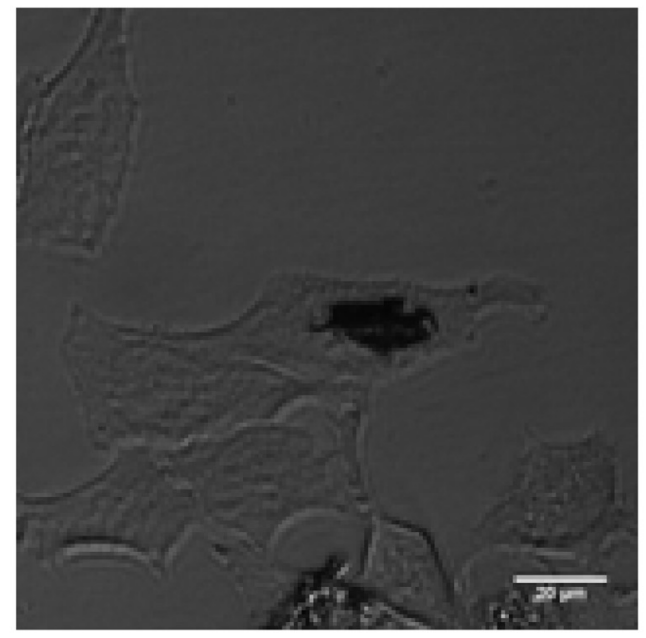

control

\section{1}

1

100 


\section{Conclusions and recommendations}

Magnetic nanowires are promising new particles for magnetic drug delivery. The FePd nanowires developed in this study show very interesting properties. Their length and diameter are tunable by choosing appropriate PCTE membranes and adjusting the deposition time and cycles. The magnetic properties of the nanowires cannot be explained in terms of superparamagnetic behaviour or pure coherent rotation. The measured magnetisation curves show remanence in all directions, but the sample is easier to saturate in the hard-axis direction $\left(90^{\circ}\right)$. This behaviour is opposite of what can be expected from available micromagnetic models. This effect might be caused by the polycrystalline properties, as is supported by TEM and XRD analysis and the increased remanence in randomly orientated nanowires.

In the biological experiments the FePd nanowires were shown to be non-cytotoxic and non-immunotoxic in the preliminary tests presented, which is essential in order to apply the particles in any in-vivo application. A magnet applicable in in-vivo animal studies was designed and optimised for trapping the FePd nanowires from capillary blood flow. The dimensions can be scaled up, while keeping the field strength and gradient sufficient for targeting, for future use in a clinical relevant application as breast tumor treatment.

In the pilot experiment no FePd nanowires could be found in the liver and kidneys of the rats indicating a low response of the immune system. Unfortunately also no significant amount of FePd nanowires could be detected in the target region. This was most likely caused by the fixation procedure in which the blood was removed from the rat and replaced by formaldehyde, which washed out the FePd wires. In future tests the fixation procedure should be avoided. Possibly a chicken embryo can be used to analyse the targeting by fluorescence without the need of fixation or removing the blood containing most of the particles.

\section{References}

[1] Y. Hirota, Y. Akiyama, Y. Izumi, S. Nishijima, Fundamental study for development magnetic drug delivery system, Physica C: Supercond. 469 (October) (2009) 1853-1856

[2] H. Xu, T. Song, X. Bao, L. Hu, Site-directed research of magnetic nanoparticles in magnetic drug targeting. J. Magn. Magn. Mater. 293 (May) (2005) 514-519.

[3] O. Mykhaylyk, N. Dudchenko, A. Dudchenko, Doxorubicin magnetic conjugate targeting upon intravenous injection into mice: high gradient magnetic field inhibits the clearance of nanoparticles from the blood, J. Magn. Magn. Mater 293 (May) (2005) 473-482.

[4] C. Alexiou, W. Arnold, R.J. Klein, F.G. Parak, P. Hulin, C. Bergemann, W. Erhardt, S. Wagenpfeil, A.S. Lübbe, Locoregional cancer treatment with magnetic drug targeting, Cancer Res. 60 (December) (2000) 6641-6648.

[5] C. Alexiou, R. Jurgons, R. Schmid, A. Hilpert, C. Bergemann, F. Parak, H. Iro, In vitro and in vivo investigations of targeted chemotherapy with magnetic nanoparticles, J. Magn. Magn. Mater. 293 (2005) 389-393.

[6] S. Goodwin, C. Peterson, C. Hoh, C. Bittner, Targeting and retention of magnetic targeted carriers (MTCs) enhancing intra-arterial chemotherapy, J. Magn. Magn. Mater. 194 (April) (1999) 132-139

[7] A.S. Lübbe, C. Bergemann, W. Huhnt, T. Fricke, H. Riess, Predinical experiences drug targeting: tolerance and efficacy, Cancer Res. 56 (1996) 4694-4701.

[8] Y. Yoshida, S. Fukui, S. Fujimoto, F. Mishima, S. Takeda, Y. Izumi, S. Ohtani, Y. Fujitani, S. Nishijima, Ex vivo investigation of magnetically targeted drug delivery system, J. Magn. Magn. Mater. 310 (March) (2007) 2880-2882.

[9] J. Dobson, Gene therapy progress and prospects: magnetic nanoparticle-based gene delivery, Gene Ther. 13 (Februar) (2006) 283-287.

[10] O.A. Pankhurst, J. Connolly, S.K. Jones, J. Dobson, Applications of magnetic nanoparticles in biomedicine, J. Phys. D: Appl. Phys. 36 (July) (2003) R167-R181.

[11] A. Hultgren, M. Tanase, C.S. Chen, G.J. Meyer, D.H. Reich, L.A. Bauer, Cell manipulation using magnetic nanowires, J. Appl. Phys. 93 (10) (2003) 7275-7280.

[12] A. Prina-Mello, Z. Diao, J.M.D. Coey, Internalization of ferromagnetic nanowires by different living cells, J. Nanobiotechnol. 4 (January) (2006) 9.
[13] A.O. Fung, V. Kapadia, E. Pierstorff, D. Ho, Y. Chen, Induction of cell death by magnetic actuation of nickel nanowires internalized by fibroblasts, J. Phys. Chem. C 112 (39) (2008) 15085-15088.

[14] F.K. Keating, M.K. Fung, D.J. Schneider, Induction of platelet white blood cell (WBC) aggregate formation by platelets and WBCs in red blood cell units, Transfusion 48 (6) (2008) 1099-1105.

[15] Qin Hu, Y. Qi Liu, N. Li, C. Cheng, S.G. Xu, N. Wang, Wei Qin, Ben Zhong Tang, Ni-NTA-Coated nanowire materials for protein enrichment and the application in a medical device used for blood glucose degradation, Nano 8 (3) (2013) 1350029.

[16] K.M. Pondman, A.W. Maijenburg, F.B. Celikkol, A.A. Pathan, U. Kishore, B. ten Haken, J.E. ten Elshof, Au coated Ni nanowires with tuneable dimensions for biomedical applications, J. Mater. Chem. B 1 (2013) 6129-6136.

[17] K. Žužek Rožman, D. Pečko, S. Šturm, U. Maver, P. Nadrah, M. Bele, S. Kobe Electrochemical synthesis and characterization of Fe 70Pd 30 nanotubes for drug-delivery applications, Mater. Chem. Phys. 133 (2012) 218-224.

[18] S. Hussain, J.A.J. Vanoirbeek, P.H.M. Hoet, Interactions of nanomaterials with the immune system, Wiley Interdiscip. Rev. Nanomed. Nanobiotechnol. 4 (2) (2012) 169-183.

[19] C. Salvador-Morales, E. Flahaut, E. Sim, J. Sloan, M.L. Green, R.B. Sim, Complement activation and protein adsorption by carbon nanotubes, Mol. Immunol. 43 (3) (2006) 193-201.

[20] C. Salvador-Morales, E.V. Basiuk, V.A. Basiuk, M.L.H. Green, R.B. Sim, Effects of covalent functionalization on the biocompatibility characteristics of multiwalled carbon nanotubes, J. Nanosci. Nanotechnol. 8 (5) (2008) 2347-2356.

[21] C. Salvador-Morales, E.V. Basiuk, V.A. Basiuk, M.L.H. Green, R.B. Sim, Effects of covalent functionalisation on the biocompatibiltiy characteristics of multiwalled carbon nanotubes, J. Nanosci. Nanotechnol. 8 (5) (2007) 2347-2356.

[22] C. Salvador-Morales, M.L.H. Green, R.B. Sim, Interaction between carbon nanotubes and biomolecules, in: E.V. Basiuk, V.A. Basiuk (Eds.), Chemistry of Carbon Nanotubes, American Scientific Publishers, Valencia, California, USA 2007 (Chapter 27).

[23] M. Rybak-Smith, K. Pondman, E. Flahaut, C. Salvador-Morales, R. Sim, Recognition of carbon nanotubes by the human innate immune system, in: R. Klingeler, R.B. Sim (Eds.), Carbon Nanotubes for Biomedical Applications, Springer Heidelberg Germany, 2011.

[24] K.M. Pondman, M. Sobik, A. Nayak, A.G. Tsolaki, A. Jäkel, E. Flahaut, S. Hampel, B. Ten Haken, R.B. Sim, U. Kishore, Complement activation by carbon nanotubes and its influence on the phagocytosis and cytokine response by macrophages, Nanomedicine: Nanotechnol. Biol. Med. 10 (March) (2014) 1287-1299.

[25] L. Sun, Y. Hao, C.L. Chien, P.C. Searson, Tuning the properties of magnetic nanowires, IBM J. Res. dev. 49 (1) (2005) 79-102.

[26] A. Aharoni, Elongated superparamagnetic particles, J. Appl. Phys. 75 (10) (1994) 5891-5893.

[27] B. Hallmark, N.J. Darton, T. James, P. Agrawal, N.K.H. Slater, Magnetic field strength requirements to capture superparamagnetic nanoparticles within capillary flow, J. Nanopart. Res. 12 (March) (2010) 2951-2965.

[28] J. Ahmadi, G. McLaughlin, Transport, Deposition, and Removal of Fine Particles: Biomedical Applications, Springer Heidelberg Germany, 2008.

29] H.C. Berg, Random Walks in Biology, 1993.

[30] V. Haehnel, S. Fähler, L. Schultz, H. Schlörb, Electrodeposition of Fe70Pd30 nanowires from a complexed ammoniumsulfosalicylic electrolyte with high stability, Electrochem. Commun. 12 (August) (2010) 1116-1119.

[31] V. Haehnel, C. Mickel, S. Fa, L. Schultz, Structure, microstructure, and magnetism of electrodeposited Fe 70 Pd 30 nanowires, J. Phys. Chem. C 114 (2010) 19278-19283.

32] H. Schlörb, V. Haehnel, M.S. Khatri, A. Srivastav, A. Kumar, L. Schultz, S. Fähler, Magnetic nanowires by electrodeposition within templates, Phys. Status Solid B 247 (August) (2010) 2364-2379.

[33] T.K. Jain, M.A. Morales, S.K. Sahoo, D.L. Leslie-Pelecky, V. Labhasetwar, Iron oxide nanoparticles for sustained delivery of anticancer agents, Mol. Pharm. 2 (3) (2005) 194-205

[34] M. Visscher, J.J. Pouw, J. Van Baarlen, J.M. Klaase, B.T. Haken, Quantitative analysis of superparamagnetic contrast agent in sentinel lymph nodes using ex vivo vibrating sample magnetometry, IEEE Trans. Biomed. Eng. 60 (2013) 2594-2602.

[35] L.A. Bauer, D.H. Reich, G.J. Meyer, Selective functionalization of two-component magnetic nanowires, Langmuir 19 (17) (2003) 7043-7048.

[36] R. Lavín, C. Gallardo, J. Palma, J. Escrig, J. Denardin, Angular dependence of the coercivity and remanence of ordered arrays of Co nanowires, J. Magn. Magn. Mater. 324 (August) (2012) 2360-2362.

[37] M. Vázquez, L.G. Vivas, Magnetization reversal in Co-base nanowire arrays, Phys. Status Solidi B 248 (October) (2011) 2368-2381.

[38] K.P. Ivanov, M.K. Kalinina, Yu.I. Levkovich, Blood flow velocity in capillaries of brain and muscles and its physiological significance, Microvasc. Res. 22 (1981) 143-155.

[39] Y.-X.J. Wang, Superparamagnetic iron oxide based MRI contrast agents: current status of clinical application, Quantitative Imag. Med. Surg. 1 (2011) $35-40$. 